\begin{tabular}{c} 
Global Journal of Mathematical Analysis, $7(1)(2019) 1-3$ \\
Global Journal of Mathematical Analysis \\
SPC \\
Website: $\begin{array}{c}\text { www.sciencepubco.com/index.php/GJMA } \\
\text { doi: } 10.14419 / \text { gima.v7il.23665 } \\
\text { Short communication }\end{array}$ \\
\hline
\end{tabular}

\title{
A new Alzer type Inequality Related to Binomial Function
}

\author{
Xiangkai Dou ${ }^{1}$ and Li Yin ${ }^{2, *}$ \\ ${ }^{1}$ Department of Mathematics, Binzhou University, Binzhou City, Shandong Province, 256603, China \\ ${ }^{2}$ Department of Mathematics, Binzhou University, Binzhou City, Shandong Province, 256603, China \\ ${ }^{*}$ Corresponding author E-mail: yinli7979@163.com
}

\begin{abstract}
In this paper, we establish a new Alzer type inequality related to binomial function by using Sitnik methods.

Keywords: binomial function; Alzer type inequality; monotonicity
\end{abstract}

]

\section{Introduction}

The difference $J_{n}(x)=e^{x}-\sum_{k=0}^{n} \frac{x^{k}}{k !}$ for real $x$ and positive integers $n$ have been studied by many mathematicians. In 1943, P. K. Menon[8] proved the intriguing inequality

$J_{n-1}(x) J_{n+1}(x)>\frac{1}{2}\left(J_{n}(x)\right)^{2}$,

which is valid for all positive integers $n$ and for all $x>0$.

Later, H. Alzer[1] established the sharpened inequality

$J_{n-1}(x) \cdot J_{n+1}(x)>\frac{n+1}{n+2}\left(J_{n}(x)\right)^{2}$,

for all $n \in \mathbf{N}$ and $x>0$, and with the best possible constant $\frac{n+1}{n+2}$.

In 2015, L. Yin and W. -Y. Cui[13] showed a generalization of Alzer inequality related to exponential function, and generalized it for the remainder of Maclaurin series.

Recently, S. M. Sitnik formulated some conjectures on monotonicity of ratios for exponential series remainders. They are equivalent to conjectures on monotonicity of a ratio of Kummer hypergeometric function, see [9] and [10]. Afterwards, K. Mehrez and S. M. Sitnik proved their conjectures in [7].

Actually, the Turán type inequalities have a more extensive literature and recently the results have been applied in problems arising from many fields such as information theory, economic theory and biophysics.For more about this subject the readers refer to [2, 3, 4, 5, 8, 6, 9, 11, 12] and the references therein.

In this paper,we consider the following binomial function

$$
\begin{aligned}
I_{n}(x) & =\frac{1}{\sqrt[p]{1+x^{p}}}-\sum_{k=0}^{n}(-1)^{k} \frac{p(p+1) \cdots(p+k-1) x^{k p}}{k !} \\
& =\sum_{k=n+1}^{\infty}(-1)^{k} \frac{p(p+1) \cdots(p+k-1) x^{k p}}{k !}
\end{aligned}
$$

where

$$
\begin{gathered}
\frac{1}{\sqrt[p]{1+x^{p}}}=1-p x^{p}+\frac{p(p+1)}{2 !} x^{2 p} \\
+\cdots+(-1)^{n} \frac{p(p+1) \cdots(p+n-1)}{n !} x^{n p}+\cdots
\end{gathered}
$$

Copyright $\odot 2016$ Xiangkai Dou and Li Yin. This is an open access article distributed under the Creative Commons Attribution License, which permits unrestricted use, distribution, and reproduction in any medium, provided the original work is properly cited. 
The main purpose of this note is to find the greatest value $C_{n, k}$, such that

$I_{n-k}(x) I_{n+k}(x)>C_{n, p, k}\left(I_{n}(x)\right)^{2}$

is valid for every positive $x$ and $n, k \in \mathbf{N}$.

We established the sharpened inequality

$\frac{I_{n-1}(z) I_{n+1}(z)}{I_{n}^{2}(z)}>\frac{(p+n+1)(n+1)}{(p+n)(n+2)}$,

and

$\frac{I_{n-k}(z) I_{n+k}(z)}{I_{n}^{2}(z)}>\frac{(p+n+1) \cdots(p+n+k)(n-k+2) \cdots(n+1)}{(p+n-k+1) \cdots(p+n)(n+2) \cdots(n+k+1)}$.

\section{2. lemmas}

Lemma 1 ([7]). Let $\left\{a_{n}\right\}$ and $\left\{b_{n}\right\},(n=0,1,2, \cdots)$ be real numbers such that $b_{n}>0$ and $\left\{\frac{a_{n}}{b_{n}}\right\}_{n \geq 0}$ is increasing(decreasing), then $\left\{\frac{a_{0}+a_{1}+\cdots+a_{n}}{b_{0}+b_{1}+\cdots+b_{n}}\right\}$ is increasing(decreasing).

Lemma 2 ([7]). Let $\left\{a_{n}\right\}$ and $\left\{b_{n}\right\},(n=0,1,2, \cdots)$ be real numbers and let the power series $A(x)=\sum_{n=0}^{\infty} a_{n} x^{n}$ and $B(x)=\sum_{n=0}^{\infty} b_{n} x^{n}$ be convergent if $|x|<r$. If $b_{n}>0,(n=0,1,2, \cdots)$ and the sequence $\left\{\frac{a_{n}}{b_{n}}\right\}_{n \geq 0}$ is (strictly)increasing(decreasing), then the function $\frac{A(x)}{B(x)}$ is also (strictly) increasing(decreasing) on $[0, r)$.

\section{3. main results}

Theorem 1. For every $n, k \in \mathbf{N}, p \geq 2$, and $0<z<1$. The function

$E(n, p, k, z)=\frac{I_{n-k}(z) I_{n+k}(z)}{I_{n}^{2}(z)}$

is strictly increasing on $(0, \infty)$. As a result, we have the following Turán type inequalities

$\frac{I_{n-k}(z) I_{n+k}(z)}{I_{n}^{2}(z)}>\frac{(p+n+1) \cdots(p+n+k)(n-k+2) \cdots(n+1)}{(p+n-k+1) \cdots(p+n)(n+2) \cdots(n+k+1)}$

where the constant $\frac{(p+n+1) \cdots(p+n+k)(n-k+2) \cdots(n+1)}{(p+n-k+1) \cdots(p+n)(n+2) \cdots(n+k+1)}$ is best possible.

Proof. simple computation yields

$$
E(n, p, k, z)=\frac{I_{n-k}(z) I_{n+k}(z)}{I_{n}^{2}(z)}
$$

$$
=\frac{\sum_{m=0}^{\infty} \sum_{j=0}^{m} \frac{(p(p+1) \cdots(p+n-k+j))(p \cdots(p+n+k+m-j))}{(n-k+1+j) !(n+k+1+m-j) !}(-1)^{2 n+2+m} z^{(2 n+2+m) p}}{\sum_{m=0}^{\infty} \sum_{j=0}^{m} \frac{(p \cdots(p+n+j))(p(p+1) \cdots(p+n+m-j))}{(n+1+j) !(n+1+m-j) !}(-1)^{2 n+2+m} z^{(2 n+2+m) p}}
$$

Define sequences $\left\{A_{n, p, k, j}\right\},\left\{B_{n, p, k, j}\right\}$ and $\left\{C_{n, p, k, j}\right\}$ by

$$
\begin{gathered}
A_{n, p, k, j}=\frac{(p \cdots(p+n-k+j))(p \cdots(p+n+k+m-j))}{(n-k+1+j) !(n+k+1+m-j) !}(-1)^{2 n+2+m}, \\
B_{n, p, k, j}=\frac{(p \cdots(p+n+j))(p \cdots(p+n+m-j))}{(n+1+j) !(n+1+m-j) !}(-1)^{2 n+2+m},
\end{gathered}
$$

and

$$
\begin{gathered}
C_{n, p, k, j}=\frac{A_{n, p, k, j}}{B_{n, p, k, j}} \\
=\frac{\frac{(p(p+1) \cdots(p+n-k+j))(p(p+1) \cdots(p+n+k+m-j))}{(n-k+1+j) !(n+k+1+m-j) !}(-1)^{2 n+2+m}}{\frac{(p(p+1) \cdots(p+n+j))(p(p+1) \cdots(p+n+m-j))}{(n+1+j) !(n+1+m-j) !}(-1)^{2 n+2+m}} \\
=\frac{(p(p+1) \cdots(p+n-k+j))}{(p(p+1) \cdots(p+n+j))} \\
\frac{(p(p+1) \cdots(p+n+k+m-j))(n+1+j) !(n+1+m-j) !}{(p(p+1) \cdots(p+n+m-j))(n-k+1+j) !(n+k+1+m-j) !} . \\
=\frac{(n+j+1-k+1) \cdots(n+j+1)}{(n+1+m-j+1) \cdots(n+1+m-j+k)}
\end{gathered}
$$




$$
\frac{(p+n+m-j+1) \cdots(p+n+m-j+k)}{(p+n+j-k+1) \cdots(p+n+j)} .
$$

Further computation results in

$$
\begin{gathered}
\frac{C_{n, p, k, j+1}}{C_{n, p, k, j}}=\frac{(p+n+m-j)(p+n+j-k+1)}{(p+n+j+1)(p+n+m-j+k)} \\
\cdot \frac{(n+2+j)(n+m-j+k+1)}{(n+j-k+2)(n+m-j+1)} \\
=\frac{\left((n+m-j)^{2}+(p+k+1)(n+m-j)+p(k+1)\right)}{\left((n+m-j)^{2}+(p+k+1)(n+m-j)+p+k\right)}
\end{gathered}
$$

$$
\cdot \frac{\left((n+j)^{2}+(p-k+3)(n+j)+2(p-k+1)\right)}{\left((n+j)^{2}+(p-k+3)(n+j)+(p+1)(2-k)\right)} .
$$

When $p \geq 2$, then $p(k+1)>p+k$, and $2(p-k+1)-(p+1)(2-k)=(p-2) k \geq 0$, so we have $\frac{C_{n, p, k, j+1}}{C_{n, p, k, j}}>1$.This implies that the sequence $C_{n, p, k, j}$ is strictly increasing to $j$. By using Lemma 2.1 and Lemma 2.2, we easily obtain the function $E(n, p, k, z)$ is strictly increasing on $(0, \infty)$.

Finally, from limit identity

$\lim _{z \rightarrow 0^{+}} \frac{I_{n-k}(z) I_{n+k}(z)}{I_{n}^{2}(z)}=\frac{(p+n+1) \cdots(p+n+k)(n-k+2) \cdots(n+1)}{(p+n-k+1) \cdots(p+n)(n+2) \cdots(n+k+1)}$,

we complete the proof.

In particular, taking $k=1$ in the Theorem 3.1, we easily obtain the following Corollary 3.1.

Corollary 1 . For every $n \in \mathbf{N}, p \geq 2$,and $0<z<1$. The function

$E(n, p, z)=\frac{I_{n-1}(z) I_{n+1}(z)}{I_{n}^{2}(z)}$

is strictly increasing on $(0, \infty)$. As a result, we have the following Turán type inequalities

$\frac{I_{n-1}(z) I_{n+1}(z)}{I_{n}^{2}(z)}>\frac{(p+n+1)(n+1)}{(p+n)(n+2)}$

where the constant $\frac{(p+n+1)(n+1)}{(p+n)(n+2)}$ is best possible.

ACKNOWLEDGEMENTS. The authors are grateful to anonymous referees for their careful corrections to and valuable comments on the original version of this paper.

The authors were supported by the Science Foundation of Binzhou University under grant BZXYL1406 and BZXYFB20150903 and by Soft Science Project of Binzhou City under 2018BRK24.

\section{References}

[1] H. Alzer, An inequality for the exponential function. Arch. Math., 55(1990),462-464.

[2] H. Alzer and G. Felder, A Turan-type inequality for the gamma function. J. Math.Anal. Appl., 350(2009), $105-109$.

[3] Á Baricz and Ponnusamy, Saminathan and Singh, Sanjeev, Turán type inequalities for general Bessel functions. Mathematics,19 2015, $709-719$.

[4] K. Dilcher, An inequality for sections ofcertain power series. Arch. Math., 60(1993),339-349.

[5] Z. Abo-Hammour, O. Abu Arqub, S. Momani, N. Shawagfeh, Optimization Solution of Troesch's and Bratu's Problems of Ordinary Type Using Novel Continuous Genetic Algorithm. Discrete Dynamics in Nature and Society, vol. 2014, Article ID 401696, 15 pages, 2014. doi.10.1155/2014/401696.

[6] M. Mehrez Turán type inequalities for the q-exponential functions. Arabian Journal of Mathematics, 6(4), (2017), 309-314.

[7] M. Mehrez and S. M. Sitnik, Proofs of some conjectures on monotonicity of ratios of Kummer, Gauss and generalized hypergeometric functions. (2014) Available online at http://arxiv.org/abs/1410.6120.

[8] P. K. Menon, Some integral inequalities. Math. Student, 11(1943),36-38.

[9] S. M. Sitnik, Conjectures on monotonicity of ratios of Kummer and Gauss hypergeometric functions. Available online at http://arxiv . org/abs/

[10] S. M. Sitnik and K. Mehrez, Proofs of some conjectures on monotonicity of ratios of Kummer, Gauss and generalized hypergeometric functions. Analysis, 36(4) 2016, 263-268.

[11] N. Shawagfeh, O. Abu Arqub, S. Momani, Analytical solution of nonlinear second-order periodic boundary value problem using reproducing kernel method. Journal of Computational Analysis Applications, 16 (2014), 750-762.

[12] M. Al-Smadi, O. Abu Arqub, N. Shawagfeh, S. Momani, Numerical investigations for systems of second-order periodic boundary value problems using reproducing kernel method. Applied Mathematics and Computation, 291 (2016), 137-148.

[13] L. Yin and W. -Y. Cui, A generalization of Alzer inequalities related to exponential function. Proceeding of Jangjeon Mathematical Society, 18(3) 2015, 385-388. 